\title{
Emulsification Properties of Lactose Fatty Acid Esters
}

\author{
Seung-Min Lee1, Ashwini Wagh ${ }^{2}$, Guneev Sandhu ${ }^{1}$, Marie K. Walsh ${ }^{*}$ \\ ${ }^{1}$ Department of Nutrition, Dietetics, and Food Sciences, Utah State University, Logan, Utah, USA \\ ${ }^{2}$ The Clorox Service Company, Pleasanton, California, USA \\ Email:marie.walsh@usu.edu
}

How to cite this paper: Lee, S.-M., Wagh, A., Sandhu, G. and Walsh, M.K. (2018) Emulsification Properties of Lactose Fatty Acid Esters. Food and Nutrition Sciences, 9, 1341-1357. https://doi.org/10.4236/fns.2018.912096

Received: November 8, 2018

Accepted: December 4, 2018

Published: December 7, 2018

Copyright (C) 2018 by authors and Scientific Research Publishing Inc. This work is licensed under the Creative Commons Attribution International License (CC BY 4.0).

http://creativecommons.org/licenses/by/4.0/

(c) (i) Open Access

\begin{abstract}
Sugar fatty acid esters (SFAE) are a class of synthetic emulsifiers used in the food, pharmaceutical, and personal care industries. The influence of the fatty acid chain length on the emulsification properties of lactose fatty acid esters (LFAE) including lactose monooctanoate (LMO), lactose monodecanoate (LMD), lactose monolaurate (LML) and lactose monomyristate (LMM) was investigated in this study. The stability of the emulsions as well as the oil droplet size distribution in $20 \%$ soybean oil-in-water emulsions was measured at $0.1 \%, 0.25 \%$ and $0.5 \%$ of LFAE concentrations. In order of LFAE with the strongest emulsion stabilization characteristics were LML, LMD, LMO and LMM. Oil droplet distributions resulted in the same trend, with LML and LMD maintaining the smallest droplet sizes and thus the most stable emulsion. The hydrophilic-lipophilic balance (HLB) and critical micelle concentrations were determined for each LFAE. An increase in HLB value was seen with an increased CMC value for each LFAE, showing the strength of the linear relationship between these two measured values. Additionally, there was a decrease in HLB and CMC values with a decrease in the fatty acid chain length of each LFAE. This research showed that LML and LMD formed more stable emulsions, even with HLB and CMC values higher than those of LMM suggesting HLB and CMC values alone do not predict emulsifier effectiveness.
\end{abstract}

\section{Keywords}

Lactose Fatty Acid Esters, Emulsions, Emulsion Stability, Sugar Esters

\section{Introduction}

The most common SFAEs consist of glucose, fructose, xylose or sucrose as the hydrophilic head group. Previous studies have investigated these esters in dif- 
ferent colloidal systems [1]-[7]. As non-ionic emulsifiers, the use of LFAEs has been recently studied in food, cosmetics and pharmaceuticals [1] [8] [9].

Studying the mechanisms by which emulsions destabilize is an important approach in understanding the emulsification properties of SFAEs. An oil-in-water $(\mathrm{O} / \mathrm{W})$ emulsion is an aqueous dispersion of oil droplets in colloidal suspension in water. These emulsions can exhibit aggregation due to the density differences between the two phases over an elapsed period of time [10] [11]. Gravitational forces play a critical role in the destabilization of emulsions [11]. The rate at which this takes place can be described mathematically using Stokes' law with considerations of Brownian movement to yield a velocity of the migration of oil droplets out of suspension and into aggregation:

$$
v=\frac{-2 g r^{2}\left(\rho^{2}-\rho^{1}\right)}{2 \eta^{1}}
$$

where $v$ is the velocity of the migrating oil (creaming), $r$ is the radius of the particle, $g$ is the acceleration due to gravity, $\rho$ is the density and $\eta$ is the shear velocity. The subscripts 1 and 2 refer to the continuous and dispersed phases. According to Stokes' law, O/W emulsions are normally stable if the oil droplet size remains at or under $1.0 \mu \mathrm{m}$ [11].

Flocculation is of practical importance as it causes the droplets to clot, leading to a growth in the mean oil droplet diameter causing droplets to coalescence and the emulsion to destabilize [11] [12]. Differences in comparative densities cause the two destabilizing phases to move toward different locations: water accumulates at the bottom, oil floats to the top, and any remaining emulsion sits in between [12]. Bands are thus formed leading to the nomenclature we use to describe these destabilized emulsions: clarification or the formation of visually clear bands due to oil and water separating and creaming or the movement of fat and oil to the top of a solution. Thus, the clarification and creaming processes indicate unstable emulsions. Emulsions are often stabilized in the long term via homogenization processes which dramatically decrease oil droplet sizes combating gravitational forces with increasing shear forces [11] [13] [14].

Clarification and creaming can be easily measured using a Turbiscan (vertical scan macroscopic analyzer) [15] [16] [17]. This light scattering detection can measure clarification and creaming in emulsions via the transmitted and backscattered light which is used to determine an emulsion stability [12]. Alternatively, many studies concluded that oil droplet size distribution within the emulsion is a better indicator of emulsion stability which can be measured with a droplet size analyzer [11] [17] [18].

Noted characteristics of SFAEs feature a high tendency to remain at the $\mathrm{O} / \mathrm{W}$ interface in an emulsion measurable by the HLB [1] [4] [5] [19]. This implies that the HLB value of an emulsion is an indicator of emulsification stability and other properties such as water solubility and CMC. It has been investigated that the SFAEs type of headgroup [1] [4] [5] [6] [7] [9] [20] and/or degree of esterification [6] can explain the variations observed in HLB and CMC values in 
emulsions made with these emulsifiers. The importance of these values, as it relates to the fatty acid chain used to generate an emulsifying ester, has been well established [1]-[7], [19] [20] [21] [22], however, there has been little attention given to SFAEs made with lactose attached to fatty acid chains and the resulting emulsifier properties of such compounds.

We were the first to report the synthesis of lactose esters [8] [23] and show the microbial inhibitory properties of lactose esters [24] [25]. The presented work investigates the emulsification properties of LMM, LML, LMO and LMD. Although there have been other reports on the emulsification properties of lactose esters [1] this research is different in that emulsion activity was determined using light scattering and oil particle size at various concentrations.

The primary objective of this research was to evaluate the emulsification ability of LMO, LMD, LML and LMM. Emulsions were prepared in $20 \%$ soybean oil and compared to commercially available emulsifiers, Tween-20 (sorbitanlaurate) and Ryoto L-1695 (sucrose monolaurate) The influence of various concentrations $(0.1 \%, 0.25 \%$ and $0.5 \%)$ over time on emulsion stability was also evaluated using both light scattering and oil particle size. Additionally, the HLB values of each emulsifier were calculated and the CMC values were determined experimentally. Evaluating the correlations between HLB and CMC to emulsion stability was done and may provide a better understanding of the emulsification properties SFAEs to emulsion stability.

\section{Materials and Methods}

\subsection{Materials and Equipment}

Soybean oil used was obtained from a local grocery store and was of the brand Western Family Inc., Madison, WI, USA. Molecular sieves 3A, vinyl octanoate, vinyl decanoate, vinyl laurate, vinyl myristate, HPLC grade solvents (acetonitrile and water), immobilized lipases from Thermomyces lanuginosus lipase enzyme (TM3), Whatman glass microfiber filters, cuvettes and EosinY were all obtained from Sigma Aldrich, St. Louis, MO, USA. Additional supplies of 2-methyl-2-butanol (2M2B) (dried using 10\% 3A molecular sieves) and Tween-20 were purchased from Thermo Fisher, MA, USA. Ryoto L-1695 was purchased from Mitsubishi-Kagaku, Tokyo, Japan and lactose was obtained from Proliant, Iowa, USA. A reversed-phased high-performance liquidchromatography (RP-HPLC) (Beckman System Gold 125 SolventModule, Ontario,

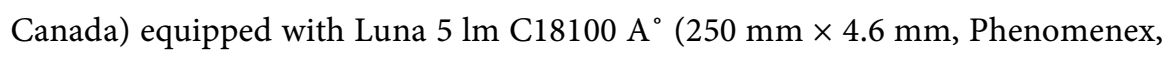
Torrance, CA, USA), an evaporative light scattering detector (ELSD) (AgilentTechnologies, Santa Clara, CA, USA), incubator shaker (Beckman, USA), Ultra-turrax T25 (Janke and Kunkel, Staufen, Germany), microfluidizer (Microfluidics Corporation, Newton, MA, USA), LS Beckman Coulter droplet size analyzer (LS 230, Coulter Corporation, Miami, FL, USA), Turbiscan (MA2000, Toulouse, France) and spectrophotometer (Beckman, Portland, OR, USA) were used. 


\subsection{Synthesis of LFAEs}

Enzymatic synthesis of LML was performed using the method as described by Walsh et al. [8]. Synthesis of LMO was conducted using lactose, vinyl octanoate, molecular sieves and TM3. For a $60 \mathrm{ml}$ reaction in 2M2B, $3 \mathrm{~g}$ of lactose, $6 \mathrm{~g}$ of dried molecular sieves, $1.7 \mathrm{ml}$ of vinyl octanoate (lactose to fatty acid ratio of 1:2.1) and $1.8 \mathrm{~g}$ TM3 were combined. The resulting mixture was placed in 100 $\mathrm{ml}$ glass bottles and then placed in the incubator/shaker and was allowed to reactat a temperature of $60^{\circ} \mathrm{C}$ while agitated by incubator/shaker at the $90 \mathrm{rpm}$ setting for 2 days. LMD and LMM were also synthesized enzymatically using the methods described above, just using different molar ratios of lactose to fatty acid and the respective substrates of vinyl myristate (lactose to fatty acid ratio of 1:2.14) for LMM and vinyl decanoate (lactose to fatty acid ratio of 1:1) for LMD.

The amount of LFAEs synthesized was determined using RP-HPLC with ELSD set at $60^{\circ} \mathrm{C}$ with a nitrogen gas pressure of 3.55 bar as previously described [8]. Once a sable baseline was obtained, the sample mixture in $2 \mathrm{M} 2 \mathrm{~B}$ is manually injected to a C18-based sorbent (stationary phase) with flow rate of $1 \mathrm{ml} / \mathrm{min}$. A linear gradient from $10 \%$ acetonitrile-water $(40: 60, \mathrm{v} / \mathrm{v})$ to $95 \%$ acetonitrile-water (95:5, v/v) over $18 \mathrm{~min}$ was used as the mobile phase [26].

\subsection{Purification of LFAEs}

For ester purification, the $2 \mathrm{M} 2 \mathrm{~B}$ reaction was filtered through a Whatman glass microfiber filter then dried in a hood for $48 \mathrm{hrs}$. The dry solids of LMM and LML were suspended in a 50\% hexane-water while the dry solids of LMO and LMD were suspended in a 50\% ethanol-water. These were then placed in a separatory funnel. The lower aqueouslayer was drained into a beaker and dried in a hood for $48 \mathrm{hrs}$. After completely drying, the product powder was suspended in hexane and/or ethanol, and then centrifuged for $15 \mathrm{~min}$ at room temperature at $2000 \times \mathrm{g}$ and the supernatant analyzed via HPLC for the presence of ditri- or higher saccharides [8]. The hexane and/or ethanol extraction was repeated until only the monoester was present in the pellet. The purities of the LFAEs were confirmed to be greater than $85 \%$ by HPLC analysis.

\subsection{HLB Calculation}

The calculated HLB ${ }^{\mathrm{a}}$ values of LFAEs, Tween-20 and Ryoto L-1695 were determined using the formula HLB $=7+\sum$ (hydrophilic values) $-\sum$ (lipophilic values) [11]. An alternative equation for $\mathrm{HLB}^{\mathrm{b}}$ calculation is the following formula: $\mathrm{HLB}=[(\mathrm{L} / \mathrm{T}) \times 20](\mathrm{L}$ is the molecular weight of the hydrophilic part of the molecule, and $\mathrm{T}$ is the total molecular weight) [27]. Superscripts were used in the data to differentiate which calculation method was used for comparative analysis.

\subsection{CMC Determination}

The CMC values of LFAEs, Tween-20 and Ryoto L-1695 were measured by the 
dye micellization method using eosin $\mathrm{Y}$ [20]. A $10 \mathrm{X}$ stock solution of the dye $(0.19 \mathrm{mM})$ was prepared in water which was diluted with water to a working standard (2X solution). Identical stock solution concentrations $(0.001 \mathrm{~g} / \mathrm{ml})$ of LFAEs were prepared in distilled water. The stock solutions were then added to each cuvette for a final concentration $0.002 \mu \mathrm{M}$ and each cuvette contained a total of $1 \mathrm{ml}$. Light absorbance was measured at $538 \mathrm{~nm}$ and $518 \mathrm{~nm}$ spectrophotometerically at emulsifier concentrations between $1 \mu \mathrm{M}$ to $1 \mathrm{mM}$, and the CMC was determined as described by Patist et al. [20]. All measurements were conducted at $25^{\circ} \mathrm{C}$.

\subsection{Emulsion Preparation}

Emulsion samples of $20 \%$ soybean oil and water were prepared by combining 40 $\mathrm{ml}$ of water and $10 \mathrm{ml}$ of soybean oil in a beaker; and each sample received one treatment of emulsifier at concentrations of $0 \%, 0.1 \%, 0.25 \%$ and $0.5 \%$ in triplicate. Each emulsifier was first stirred in the $40 \mathrm{ml}$ of water for $15 \mathrm{~min}$ before the addition of the $10 \mathrm{ml}$ of oil. The water and oil phases were mixed with a high-speed blender at 18,000 rpm for $5 \mathrm{~min}$ and then passed immediately through a microfluidizer three times at $17.4 \pm 1.6 \mathrm{MPa}(\sim 15,000 \mathrm{psi})$. All emulsion samples were prepared intriplicate. Emulsion destabilization measured by light scattering and oil droplet size measurements started on day 0 (the day the emulsions were prepared) and continued daily until day 4 with emulsions stored at room temperature. All emulsions were prepared and analyzed in triplicate and the standard errors were calculated.

\subsection{Emulsion Stability Measurements}

The stability of each $20 \%$ oil-in-water $(\mathrm{O} / \mathrm{W})$ sample prepared as described above was determined using a Turbiscan, a vertical scan macroscopic analyzer, with Turbiscan MA2000 software. Samples were prepared by placing $5 \mathrm{ml}$ of each sample into $11 \mathrm{~cm}$ glass tubes. Light scattering was measured daily and the thickness (in $\mathrm{mm}$ ) of the clarification layer at the bottom of the tubes over the course of the 5 days was calculated as described by Garg et al. [17]. The destabilization thickness in $\mathrm{mm}$ for each sample was plotted against the number of days. The resulting slope of the scatter plot was used to determine instability of emulsions in $\mathrm{mm} / \mathrm{d}$ and the average of the triplicate samples was calculated.

The oil droplet diameter distribution of the emulsion samples was measured using a LS Beckman Coulter droplet size analyzer (LS 230) with the polarization intensity differential set for scanning small fluid modules. Emulsions containing were analyzed from day 0 to day 4 for change in droplet diameter over time. The oil droplet measurements were taken by the angular dependence of the intensity of the laser light $(\lambda=623.8 \mathrm{~nm})$ scattered by emulsions as described by Garg et al. [17]. Droplet diameter curves were constructed as a function of the total volume of oil droplets as a percentage ( $y$-axis) versus droplet diameters (x-axis). 


\section{Results}

\subsection{Emulsion Destabilization}

Destabilization of emulsions can be observed through changes in the light backscattering properties of the emulsions. In destabilized emulsions multiple layers can develop including an oil layer on top, a creaming layer directly underneath, and clearing layer (clarification layer) at the bottom. Using a vertical scan macroscopic emulsion in glass tubes were measured along the length of the tube for changes in backscattering. Measurements were collected, and statistical analysis was used to determine the change thickness of the clarification layer $(0-10 \mathrm{~mm})$ from the bottom of each tube. Figure 1 shows how, after 5 days, the emulsions' clarification layer changes in emulsions with and without emulsifier. In Figure 1 (a), a control sample of $20 \% \mathrm{O} / \mathrm{W}$ emulsion is shown and a destabilized result was observed with a thin oil layer on top and a clarification layer on the bottom after 5 days. Conversely, Figure 1 (b), is a $20 \% \mathrm{O} / \mathrm{W}$ emulsion containing $0.5 \%$ LML after 5 days and has no visible oil or clarification layers.

Figure 2 shows the change in destabilization $(\mathrm{mm}$ ) (as an increase in the clarification layer) over the 5 days tested for $20 \% \mathrm{O} / \mathrm{W}$ emulsions containing the four synthesized LFAEs and two commercial non-ionic emulsifiers (Tween-20 and Ryoto L-1695). By measuring the slope of the increasing size of the clarification layer, we can determine the rate of destabilization; which, by definition for a stable emulsion, is less than $1.0 \mathrm{~mm} / \mathrm{d}$ [11]. Three different levels $(0.1 \%, 0.25 \%$ and $0.5 \%$ ) of each emulsifier were compared to a negative control, the oil and water mixture without an emulsifier which had a destabilization value of 3.91 $\mathrm{mm} / \mathrm{d}$. In this study, the average destabilization rates of emulsion stabilized with $0.5 \%, 0.25 \%$ and $0.1 \%$ of Tween-20 were $0.5,0.83$ and $1.7 \mathrm{~mm} / \mathrm{d}$, respectively (Figure 2(e)). This shows that the destabilization rates of Tween-20 emulsions

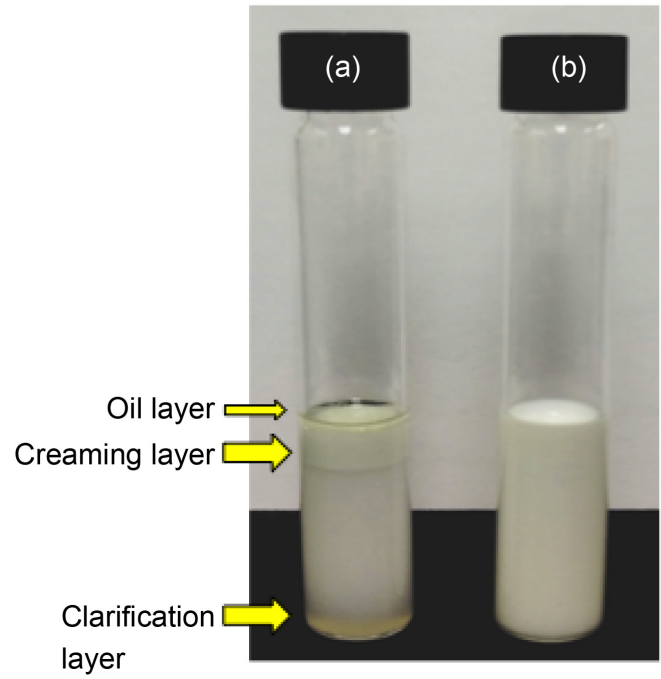

Figure 1. Stability of $20 \%$ soybean $\mathrm{O} / \mathrm{W}$ emulsion at $25^{\circ} \mathrm{C}$ after 5 days. (a) No emulsifier used. (b) The emulsion prepared with $0.5 \%$ LML. 


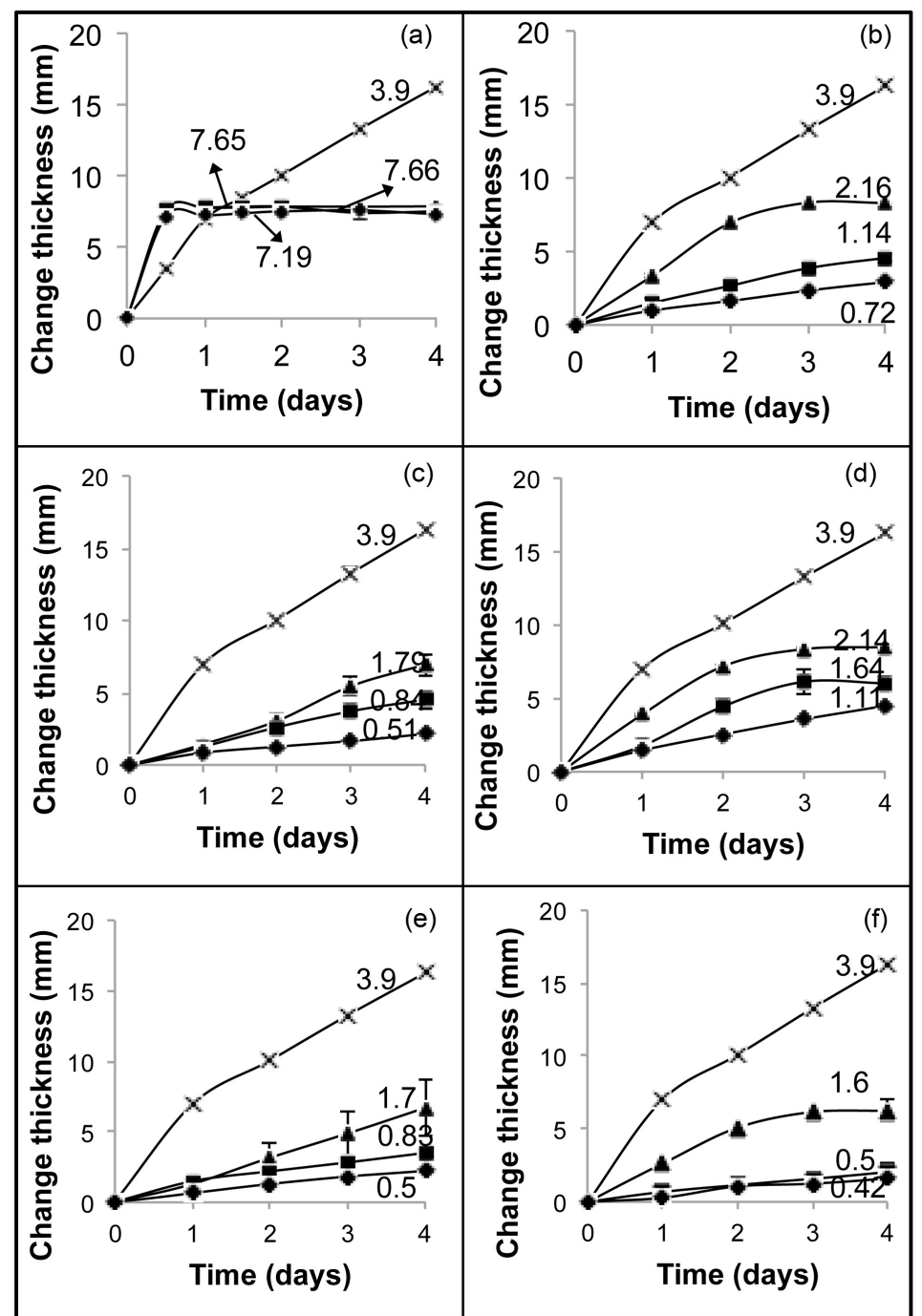

Figure 2. Change in thickness $(\mu \mathrm{m})$ of clarification layer of the emulsions at $25^{\circ} \mathrm{C}$. The change in thickness of the clarification layer (at the bottom of the tube from $0.5-10 \mathrm{~mm}$ ) in emulsions formulated with (a) LMO; (b) LMD; (c) LML; (d) LMM; (e) Tween-20; (f) Ryoto L-1695 at concentrations of $(\mathrm{X}) 0 \%(\boldsymbol{\Delta}) 0.1 \%,(-) 0.25 \%$ and $(\bullet) 0.5 \%$ over 5 days. The error bars indicate the standard errors.

are proportionate to the Tween-20 concentration. Emulsification ability of other LFAEs can be measured relative to Tween-20.

LMO stabilized emulsions were highly unstable at $0.5 \%, 0.25 \%$ and $0.1 \%$ tested with a destabilization rate of $7.19,7.65$ and $7.66 \mathrm{~mm} / \mathrm{d}$, respectively (Figure 2(a)). In order to get the observed initial destabilization slope in LMO treated emulsions, additional testing was preformed every $12 \mathrm{hrs}$ to quantify the rapid destabilization of these emulsions. LMO fails the desired level of less than $1.0 \mathrm{~mm} / \mathrm{d}$, which has been defined as an emulsifier with effective stabilization ability.

The emulsions containing LMD at $0.5 \%$ concentration produced the destabilization rate of $0.72 \mathrm{~mm} / \mathrm{d}$, implying this emulsion was stable over the time 
frame (Figure 2(b)). However, with decreased concentrations, such as $0.25 \%$ and $0.1 \%$, LMD emulsions became less stable with destabilization rates of 1.14 and $2.16 \mathrm{~mm} / \mathrm{d}$, respectively, showing less than the $1.0 \mathrm{~mm} / \mathrm{d}$ destabilization required to be an effective emulsifier.

In Figure 2(c), the average destabilization rates for the LML at concentrations of $0.5 \%, 0.25 \%$ and $0.1 \%$ were $0.51,0.84$ and 1.79 , respectively. LML at concentrations of $0.5 \%$ and $0.25 \%$ were successful at stabilizing emulsions, showing less than the $1.0 \mathrm{~mm} / \mathrm{d}$ destabilization, and thus formed effective emulsions although the emulsions formed using $0.1 \%$ LML were not stable. The emulsions containing Ryoto L-1695 (sucrose monolaurate) (Figure $2(\mathrm{f})$ ) at $0.5 \%$ and $0.25 \%$ concentrations produced a destabilization rate of 0.42 and $0.5 \mathrm{~mm} / \mathrm{d}$ respectively, implying these emulsions were stable over the time frame, similar to LML.

LMM produced less stable emulsions which decreased in stability as the concentration decreased (Figure 2(d)). Destabilization rates of 1.11, 1.64 and 2.14 $\mathrm{mm} / \mathrm{d}$ at $0.5 \%, 0.25 \%$ and $0.1 \%$ LMM concentrations, respectively were obtained. Therefore, LMM is not a suitable $\mathrm{O} / \mathrm{W}$ emulsifier, based on the destabilization rates.

\subsection{Oil Droplet Size Distribution and Measurement}

The stability of the emulsion for each LFAE was also studied by measuring the oil droplet size distribution of each emulsion over 5 days. Figure 3 shows the droplet size distribution, as a percent of droplets at a certain volume of the emulsions at $0.5 \%$ concentration of emulsifier over time. The stability of an emulsion is influenced by flocculation and/or coalescence and stable emulsions generally have small oil droplets [11] [28]. Using a high-speed blender, oil droplets sizes ranging between 2 and $10 \mu \mathrm{m}$ can be produced [11]. Even smaller oil droplets, less than $0.1 \mu \mathrm{m}$, can be generated by microfluidization [11]. In this study, high-speed blending followed by microfluidization was used to test emulsion stability as influenced by oil droplet size.

In Figure $3(\mathrm{e})$ the $20 \% \mathrm{O} / \mathrm{W}$ emulsions stabilized by $0.5 \%$ Tween- 20 , the positive control, on day 0 shows a monomodal size distribution, with a peak at $15 \%$ of the oil droplets in the range of $0.8-2 \mu \mathrm{m}$. After 2 days, a peak at $13 \%$ of the oil droplets remained in this range, and this droplet size distribution stayed relatively constant over 5 days. Additionally, these peaks stayed narrow, with a small range of distribution, without bimodality developing over time. This demonstrates an effective emulsifier.

Emulsions containing $0.5 \%$ LMO on day 0 show a bimodal drop size distribution, which peaked at $10 \%$ of oil droplets in the range of $0.5-1 \mu \mathrm{m}$ and peaked again at $7 \%$ of the droplets in the range of $1-4 \mu \mathrm{m}$ (Figure 3(a)). This oil droplet size distribution on day 2 remained in the same range. After 4 days, the droplet diameters increased to $0.5-10 \mu \mathrm{m}$, and the droplet size distribution became wider and skewed to the right, meaning the population of large oil droplets sizes became the greater percentage of total oil droplets in the emulsion. 


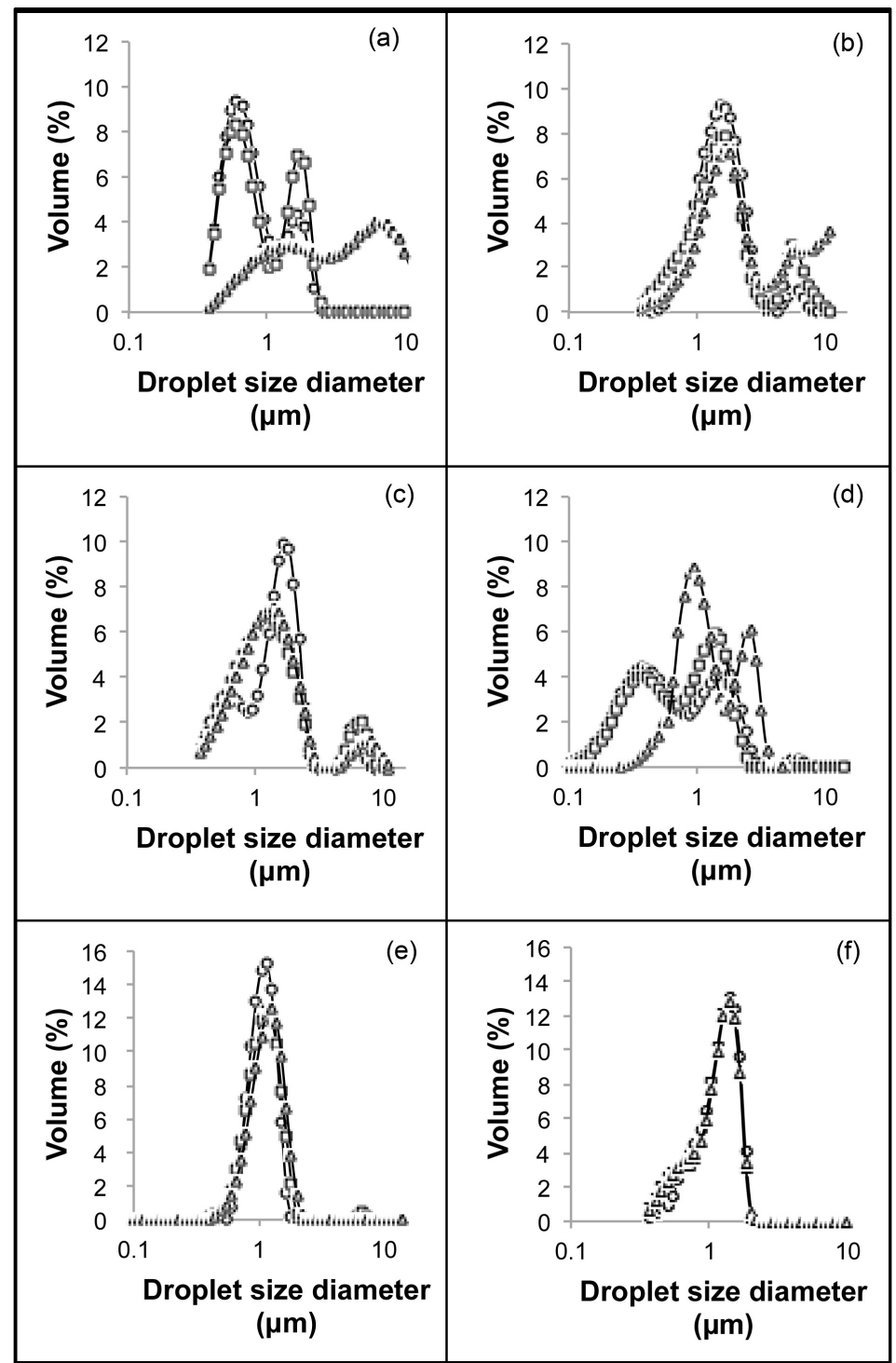

Figure 3. Oil droplet size diameter $(\mu \mathrm{m})$ distribution with volume (\%) of the emulsions at $25^{\circ} \mathrm{C}$. Droplet size diameter distribution with respect to percentage of the volume at (a) $0.5 \%$ LMO. (b) $0.5 \%$ LMD. (c) $0.5 \% \mathrm{LML}$. (d) $0.5 \%$ LMM. (e).

In Figure 3(b), the emulsion containing 0.5\% LMD on day 0 has a peak of $9 \%$ of oil droplets in the range of $1-3 \mu \mathrm{m}$ and another peak of $0.5 \%$ in the range of $6-10 \mu \mathrm{m}$. On day 2 , the smaller oil droplet sizes kept a similar distribution, but the $6-10 \mu \mathrm{m}$ range of peaks increased to $3 \%$. At day 4 , this trend continued with a bimodal skew to larger oil droplets.

At day 0 , the $0.5 \%$ LML stabilized emulsion had a significant population of droplets in the $0.5-5 \mu \mathrm{m}$ range peaking at $7 \%$ with a second right skewed distribution peak at $1 \%$ in the range of $8-10 \mu \mathrm{m}$ Figure $3(\mathrm{c})$. On day 2, the $0.5-5$ $\mu \mathrm{m}$ ranged peak increased to $10 \%$ of droplets and the second peak increased to $2 \%$ while widening its range to $5-10 \mu \mathrm{m}$. This same droplet size distribution remained on day 4 . At day 0 , the $0.5 \%$ Ryoto L- 1695 stabilized emulsion had a 
significant population of droplets in the $0.5-3 \mu \mathrm{m}$ range peaking at $13 \%$, and this droplet size distribution stayed relatively constant over 5 days (Figure 3(f)).

Emulsions with 0.5\% LMM exhibited noticeable destabilization during the 5 days of storage (Figure 3(d)). LMM emulsions were characterized by broad droplet size distributions with large bimodal peaks. On day 0 and day 2, there were a significant number of smaller droplets ranging from $0.1-1 \mu \mathrm{m}$. By day 4 , this $0.1-1 \mu \mathrm{m}$ range disappeared and droplet size was shifted to higher values, though bimodality remained. A peak of $9 \%$ of droplets was in the range of 0.5 - 3 $\mu \mathrm{m}$ and a peak of $6 \%$ of the droplets was in the range of $3-10 \mu \mathrm{m}$.

\subsection{Surface-Active Properties}

The values of MW, HLB, CMC (mM), and CMC (mM reported from other sources) for each LFAE, Tween-20 and Ryoto L-1695 are presented in Table 1. The MW of Tween-20 with an ethoxylated sorbitan was greater than Ryoto L-1695 and the LFAEs (which where themselves comparable, only differentiated by their different bound fatty acid chains). The HLB scale is a basic indicator of an emulsifier's solubility and is the primary criteria for selecting an emulsifier in a food system [22]. Typically, the maximum HLB value of non-ionic emulsifiers is 20 [11] [27]; with emulsifiers with HLB values ranging from 8 to 18 are generally used in $\mathrm{O} / \mathrm{W}$ emulsions [11] [29]. The results in Table 1 show that Tween-20, Ryoto L-1695, and all tested LFAEs are greater than 8 but less than 18 , thus all show potential at stabilizing $20 \% \mathrm{O} / \mathrm{W}$ emulsions. All the HLB ${ }^{\mathrm{a}}$ values of the tested emulsifiers are between 14.1 and 16.9 as determined by the McClements equation [11]. Using an alternative equation for $\mathrm{HLB}^{\mathrm{b}}$ calculation [24], we still find a very similar HLB value range of 13.4 to 15.8 .

Table 1. Emulsifier properties.

\begin{tabular}{|c|c|c|c|c|c|c|}
\hline Emulsifier & Composition & $\mathrm{MW}^{1}$ & $\mathrm{HLB}^{\mathrm{a} 2}$ & $\mathrm{HLB}^{\mathrm{b} 3}$ & $\begin{array}{l}\mathrm{CMC}^{4} \\
(\mathrm{mM})\end{array}$ & $\begin{array}{c}\text { Reported } \\
\text { CMC (mM) }\end{array}$ \\
\hline Tween-20 & $\begin{array}{l}\text { Sorbitan laurate } \\
\qquad\left(\mathrm{C}_{26} \mathrm{H}_{50} \mathrm{O}_{10}\right)\end{array}$ & 1227.5 & 16.7 & 15.6 & 0.07 & $0.042^{5}$ \\
\hline $\begin{array}{c}\text { Ryoto } \\
\text { L-1695 }\end{array}$ & $\begin{array}{l}\text { Sucrose monolaurate } \\
\qquad\left(\mathrm{C}_{24} \mathrm{H}_{44} \mathrm{O}_{12}\right)\end{array}$ & 524 & 16 & 15 & 0.44 & $0.42^{6}$ \\
\hline LMO & $\begin{array}{l}\text { Lactose monooctanoate } \\
\qquad\left(\mathrm{C}_{20} \mathrm{H}_{36} \mathrm{O}_{12}\right)\end{array}$ & 468.54 & 16.9 & 15.8 & 0.96 & $0.76^{6}$ \\
\hline LMD & $\begin{array}{l}\text { Lactose monodecanoate } \\
\qquad\left(\mathrm{C}_{22} \mathrm{H}_{40} \mathrm{O}_{12}\right)\end{array}$ & 496.55 & 16 & 14.8 & 0.89 & $0.56^{6}$ \\
\hline LML & $\begin{array}{l}\text { Lactose monolaurate } \\
\qquad\left(\mathrm{C}_{24} \mathrm{H}_{44} \mathrm{O}_{12}\right)\end{array}$ & 524.28 & 15.7 & 14.1 & 0.72 & $0.31^{6}$ \\
\hline LMM & $\begin{array}{l}\text { Lactose monomyristate } \\
\qquad\left(\mathrm{C}_{26} \mathrm{H}_{48} \mathrm{O}_{12}\right)\end{array}$ & 552.33 & 14.1 & 13.4 & 0.56 & $\mathrm{NR}^{7}$ \\
\hline
\end{tabular}

${ }^{1} \mathrm{MW}$, molecular weight. ${ }^{2} \mathrm{HLB}^{\mathrm{a}}$, hydrophile-lipophile balance $=\mathrm{HLB}=7+\sum$ (hydrophilic values) $\sum$ (lipophilic values). (McClements, 2005). ${ }^{3} \mathrm{HLB}^{\mathrm{b}}$, hydrophile-lipophile balance $=[(\mathrm{L} / \mathrm{T}) * 20]$. (Ritthitham (2009). ${ }^{4} \mathrm{CMC}$, critical micelle concentration. ${ }^{5}$ Reported in [20]. ${ }^{6}$ Reported in [1]. ${ }^{7}$ Not reported. 
Like HLB values, CMC values are parameters used to characterize the potential of emulsifiers [1] [26]. As such, the CMC values of the commercial emulsifiers and the LFAEs, as measured by the dye micellization method, were obtained (Table 1). Of the compounds tested, Tween-20 and Ryoto L-1695 had lower CMC values, $0.07 \mathrm{mM}$ and $0.44 \mathrm{mM}$, respectively, than the LFAEs. LMO, LMD, LML, and LMM had CMC values of $0.96 \mathrm{mM}, 0.89 \mathrm{mM}, 0.72 \mathrm{mM}$ and $0.56 \mathrm{mM}$, respectively. These results would normally suggest that these LFAEs would be expected to underperform as emulsifiers when compared to Tween-20. These experimental results on CMC values closely agree with other reported CMC values except for the values for LML. Patist et al. used the same dye micellization method with Tween-20 for the reported CMC value of $0.042 \mathrm{mM}$ [20]. Using a surface tension method, Zhang et al. determined CMC values of Ryoto L-1695, LMO, LMD and LML at values below the CMC values found in this research [1]. It is worth noting that the surface tension method, if used on impure samples, would yield a lower CMC than the dye micellization method. As the surface tension method is very sensitive to the presence of impurities [20].

An observed increase in CMC values was seen along with an increased HLB values showing the strength of the relationship between these two (Figure 4(a)) with a $R^{2}$ value of 0.975 . There is also an increase in both HLB and CMC values with a decrease in fatty acid chain lengths in Figure $4(b)\left(R^{2}=0.99\right)$ and Figure $4(c)\left(R^{2}=0.97\right)$, respectively. This shows a linear relationship between HLB and $\mathrm{CMC}$ values related to fatty acid chain length.

\section{Discussion}

Destabilization of emulsions was observed through the change in thickness of the clarification layer of the $20 \% \mathrm{O} / \mathrm{W}$ emulsions for LFAEs compared to controls. Tween-20, a sorbitanmonolaurate, is a non-ionic detergent approved by the U.S. FDA for food use [30]. Due to its wide current usage in industry and its' relative similarity to the compounds tested in this study, Tween-20 was selected as a control for comparative testing. Ryoto L-1695, a sucrose ester, also has several physical similarities to LFAEs and so was selected as another control for comparative analysis. Out of the 4 compounds tested, LML at a concentration of $0.5 \%$ was the only LFAE to exert an emulsifying activity comparable to Tween-20 and Ryoto L-1695 over 5 days. Even though LML produced less stable emulsions as the concentration decreased, Tween-20 and Ryoto L-1695 showed similar behaviors at lower concentrations.

While there are a large variety of properties we can use to characterize the emulsification stabilities of LFAEs, the HLB values can give an insight into emulsion stabilization effectiveness. A well-balanced ratio of hydrophilic head to hydrophobic tail allows for oil droplets to be stabilized by the emulsifier resulting in low destabilization rates.

The results are consistent with the limited number of studies which have investigated the HLB values of SFAEs which concluded that SFAEs made with laurate were better emulsifiers with greater emulsion stability than alternative 


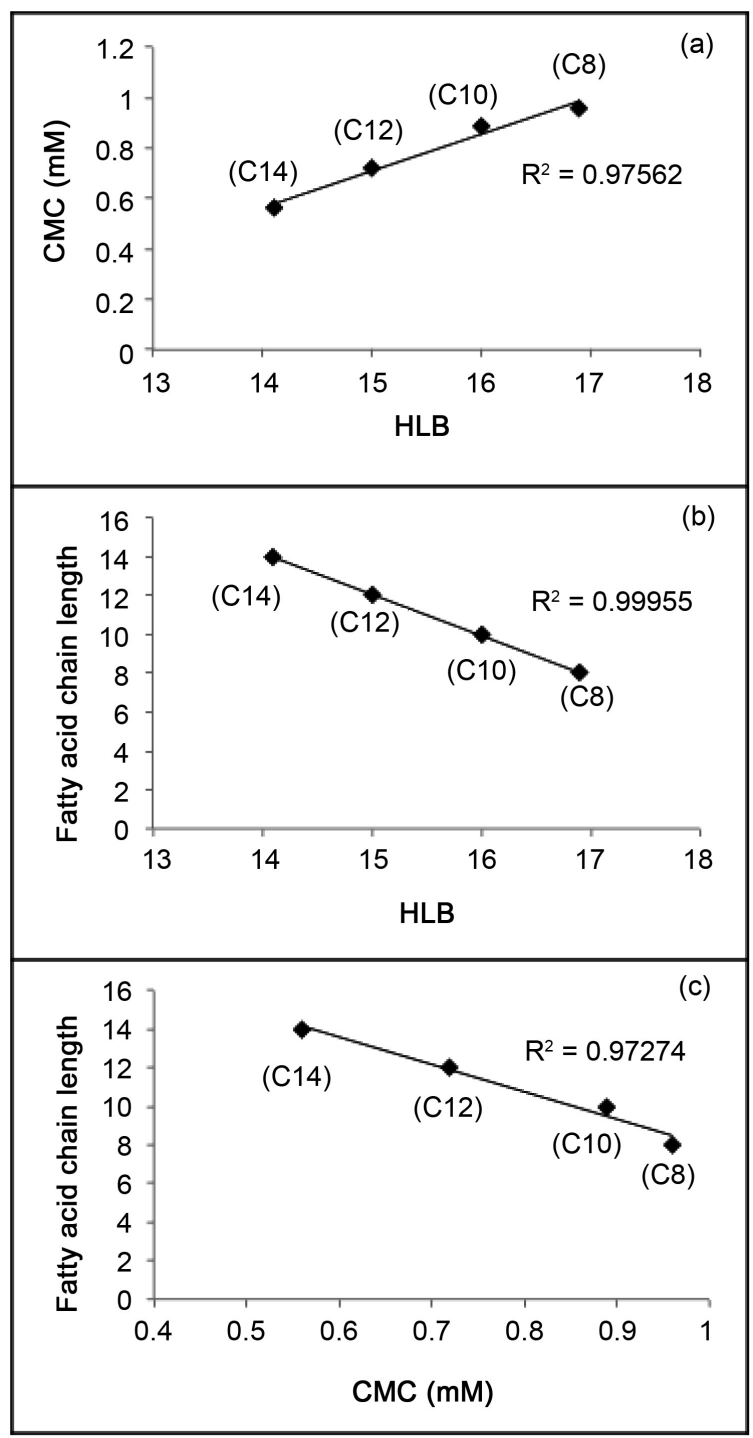

Figure 4. Comparison of HLB, CMC values and fatty acid chain length of lactose esters. Influence of the chain length of the fatty acid ester on the HLB and CMC value at $25^{\circ} \mathrm{C}$, for lactose esters containing octanoate (C8), decanoate $(\mathrm{C} 10)$, laurate $(\mathrm{C} 12)$ and myristate $(\mathrm{C} 14)$. (a) CMC vs HLB. (b) Fatty acid chain length vs HLB. (c) Fatty acid chain length vs CMC.

length fatty acid chains. Piao and Adachi reported that erythritol esterified with lauric acid (HLB; 16) resulted in more stable emulsions than lauric esters made with glycerol, arabitol, ribitol and xylitol at $0.05 \%$ [4]. Zhang et al. prepared sugar monoesters including sucrose, maltose, lactose with fatty acid chain lengths from 8 to 12 and showed that lauryl (HLB; 13.1) esters were significantly better at stabilizing emulsions compared to octanoyl (HLB; 14.5) and decanoyl (HLB; 13.8) esters in $20 \% \mathrm{O} / \mathrm{W}$ emulsions [1]. These data demonstrated that the HLB values of emulsifiers influenced the stability of emulsions.

The importance of the HLB values as it relates to the fatty acid chain used to 
generate an emulsifying ester was investigated in this study. It was evident that as LML was the most effective emulsifier tested. While the best result was still LML, LMD showed the second lowest emulsion destabilization rates followed by LMM and LMO, respectively. This bring up an interesting point, the tested LFAEs had comparable HLB values and yet very different emulsification properties. This does suggest that HLB is not the only property influencing emulsification potential.

The $0.5 \%$ LML stabilized emulsion had a significant population of droplets in the 0.5 - $5 \mu \mathrm{m}$ range. Correspondingly, Tween-20 and Ryoto L-1695 showed emulsification properties at concentrations of $0.5 \%$ with an oil droplet distribution range of $0.8-2 \mu \mathrm{m}$ and $0.5-2 \mu \mathrm{m}$, respectively. Emulsifying activity effects of Tween-20 were observed in another study in which oil droplet sizes decreases from $25 \mu \mathrm{m}$ to $150 \mathrm{~nm}$ as the concentration of the emulsifier increased from $1 \%$ to $4 \%$; oil droplet sizes then remained constant on further Tween-20 addition up to $5 \%$ [31]. Emulsions containing $0.5 \%$ LML produced narrow distributions in ranges comparable to Tween-20 and Ryoto L-1695, though bimodality was present and range distributions widened over time, while the commercial emulsifiers were consistent over time. However, these widening distributions shifted left, suggesting oil droplets were decreasing in size as time progressed. Moreover, the bimodality was minor; with the large peak remaining small and showing the same left shifting change observed in the primary peak. Thus, emulsion destabilization data shows LML is comparable with the commercial emulsifiers tested, even with the differences in oil droplet diameters and LML's bimodality. It is also interesting to consider that LML at the $0.5 \%$ concentration seems to be decreasing oil droplet sizes with only a factor of advancing time, a trend that may increase emulsion stability and delay phase separation.

Not all LFAEs yielded consistent emulsion stability profiles. LMO and LMM at $0.5 \%$ generated emulsions with oil droplet dispersions that changed greatly over time. Emulsions made with $0.5 \%$ LMD initially produced a bimodal oil droplet distribution with a large narrow peak in a range similar to LML. As time elapsed, however, the droplet size distributions became wider and even more bimodal due to increased coalescence of the smallest oil droplets. LMD stabilized emulsions followed a similar droplet size distribution as LML over 5 days albeit more exaggerated, resulting in stable emulsions, though not as stable as those made with Tween-20 and Ryoto L-1695. Although LMM at a $0.5 \%$ concentration initially showed a large portion of very small oil droplet diameters of $\leq 0.5 \mu \mathrm{m}$, a bimodal oil droplet distribution remained over time. LMO also showed small oil droplets and bimodality. LMO, though, had a very large increase in the proportion of oil droplets between 0.5 and $10 \mu$ mat $0.5 \%$ concentration during the course of the test. There was also a clear bimodal or multi-modal oil droplet size distribution growing toward larger droplets suggesting coalescence over time. This might be an indicator of the lower hydrophobic moiety of LMO compared to its hydrophilic moiety which may result in unfavorable absorption on the 
$\mathrm{O} / \mathrm{W}$ interface leading to a loss of stability over time. These similarities suggest that while the HLB values of LMO and LMM are similar considering a range of HLB values of $8-18$ are suitable to stabilize O/W emulsions, they do not accurately indicate the ability of each LFAE to interface at the oil and water surfaces of oil droplets effectively. It is true that LMM and LMO stabilized emulsions did contain small oil droplets initially, however, as previously reported in the destabilization rates study, LMM was not an effective emulsifier, being surpassed in all trials by LMD and LML.

Another explanation of the bimodal oil size distribution in the observed results for LML (along with other LFAEs tested) might be an artifact of oil droplets flocculating and/or coalescing. However, the former is unlikely due to the nature of the droplet size analyzer which makes use of agitation to spread out individual oil droplets for analysis. Furthermore, as $20 \%$ O/W emulsions are continually being diluted, thus flocculated oil droplets would be dispersed, breaking the flocculate; single coalesced oil droplets would thus be the dominant nature of droplets.

As part of this research, we investigated the effectiveness of CMC values for estimating initial emulsifier potential for LFAEs. Of the esterstested, Ryoto L-1695 and LML which have different sugar headgroups for a fixed hydrophobic group, had CMC values of $0.44 \mathrm{mM}$ and $0.72 \mathrm{mM}$, respectively. This difference is expected, as typically the hydrophobicity of the sugar headgroup of LFAEs can be considered to explain the variations observed in the CMC and HLB values as well. Giulietti and Bernardo reported that the sugars in order of highest solubility in water were fructose, sucrose, glucose followed by lactose [32]. Emulsifiers having greater hydrophobicity are theoretically more surface-active and thus facilitate micelle formation, exhibiting low $\mathrm{CMC}$ values and greater emulsification properties [33 s]. Lactose is the least hydrophilic than the other mentioned sugars and may form more stable emulsions via its better ability to influence surface-interface stability. Garofalakis et al. reported that emulsifiers with more hydrophobic headgroups, such as lactose, exhibited better surface activity than those with greater hydrophilic headgroups, such as sucrose [7]. However, in our study, LML showed a higher CMC value than Ryoto L-1695, which has sucrose as its headgroup. Zhang et al. reported CMC values of LFAEs containing decanoate and laurate as slightly above the CMC values of Ryoto L-1695 (Table 1) [1]. Thus, we need to look at the effectiveness of the fatty acid chain lengths with lactose and if the resulting emulsification properties are correlated to the CMC values.

This study clearly shows that the increasing chain lengths of the fatty acids in the respective esters significantly influences the HLB values with a decrease in HLB value due to increasing hydrophobicity. With HLB decreasing, the CMC also decreased with the increasing hydrophobicity resulting from the fatty acid chain lengths for a fixed hydrophilic group. As expected, LMM showed lower HLB and CMC values than LMO, LMD and LML since it has a longer fatty acid 
chain length. Yanke et al. demonstrated that as fatty acid chain lengths were increased, sucrose monopalmitate was more hydrophobic than sucrose monolaurate, exhibiting lower CMC values [3]. It stands to reason that in a like manner the hydrophobicity of LMM should make it a better $20 \% \mathrm{O} / \mathrm{W}$ emulsifier than the other LFAEs, as it should be more likely to be adsorbed at the O/W interface, resulting in lower CMC values as compared with LMO, LMD and LML. However, LMD and LML formed more stable emulsions, even with HLB values higher than those of LMM which would normally suggest poorer performance. The implication from these findings is that while HLB and CMC values may be an important characteristic for evaluating the activity of a non-ionic emulsifier; HLB and $\mathrm{CMC}$ values do not assure suitability for a specific application as an emulsifier. It may be that HLB and CMC values do not adequately describe the careful balance of hydrophobicity to hydrophilicity that truly dictate emulsifiers effectiveness at emulsion stability.

\section{Conclusion}

Results suggest that the chain length of the fatty acid ester significantly influences the emulsification properties of LFAEs in $20 \%$ soybean $\mathrm{O} / \mathrm{W}$ emulsions. While Tween-20 and Ryoto L-1695 are suitable at forming and stabilizing initial emulsions, LML may actually be actively working to increase emulsion stability over time in addition to its initial stabilization. LMD, with such similar physical properties, may also be an effective emulsifier in spite of its observed bimodal distribution of oil droplet sizes. It is feasible that the CMC values of LMM indicate favorable absorption during emulsification which leads to positive interactions with the oil droplets and the water phase, however, just because an emulsifier has a suitable CMC value that does not guarantee its appropriateness for a specific application as an emulsifier. It will be interesting to see how LML and LMD behave as emulsifiers in a salad dressing, or other food systems at the concentrations tested in this research. Future research can also compare these two LFAEs to other commonly used food grade non-ionic emulsifiers to determine actually viability of use.

\section{Acknowledgements}

This project was partially supported by the Utah State University Utah Agricultural Experiment Station and approved as journal paper number 9115.

\section{Conflicts of Interest}

The authors declare no conflicts of interest.

\section{References}

[1] Zhang, X., Song, F., Taxipalati, M., Wei, W. and Feng, F. (2014) Comparative Study of Surface-Active Properties and Antimicrobial Activities of Disaccharide Monoesters. PLoS ONE, 9, e114845. https://doi.org/10.1371/journal.pone.0114845

[2] Becerra, N., Toro, C., Zanocco, A.L., Lemp, E. and Günther, G. (2008) Characteri- 
zation of Micelles Formed by Sucrose 6-O-Monoesters. Colloids and Surfaces A: Physicochemical and Engineering Aspects, 327, 134-139.

https://doi.org/10.1016/j.colsurfa.2008.06.012

[3] Yanke, L., Shufen, Z., Qinghui, W. and Jinzong, Y. (2004) Relationship of Solubility Parameters to Interfacial Properties of Sucrose Esters. Colloids and Surfaces A: Physicochemical and Engineering Aspects, 248, 127-133. https://doi.org/10.1016/j.colsurfa.2004.07.032

[4] Piao, J. and Adachi, S. (2006) Stability of O/W Emulsions Prepared Using Various Monoacyl Sugar Alcohols as an Emulsifier. Innovative Food Science \& Emerging Technologies, 7, 211-216. https://doi.org/10.1016/j.ifset.2006.04.002

[5] Soultani, S., Ognier, S., Engasser, J.-M. and Ghoul, M. (2003) Comparative Study of Some Surface Active Properties of Fructose Esters and Commercial Sucrose Esters. Colloids and Surfaces A: Physicochemical and Engineering Aspects, 227, 35-44. https://doi.org/10.1016/S0927-7757(03)00360-1

[6] Ferrer, M., Comelles, F., Plou, F.J., Cruces, M.A., Fuentes, G., Parra, J.L. and Ballesteros, A. (2002) Comparative Surface Activities of Di- and Trisaccharide Fatty Acid Esters. Langmuir, 18, 667-673. https://doi.org/10.1021/la010727g

[7] Garofalakis, G., Murray, B.S. and Sarney, D.B. (2000) Surface Activity and Critical Aggregation Concentration of Pure Sugar Esters with Different Sugar Headgroups. Journal of Colloid Interface Science, 229, 391-398. https://doi.org/10.1006/jcis.2000.7035

[8] Walsh, M.K., Bombyk, R.A., Wagh, A., Bingham, A. and Berreau, L.M. (2009) Synthesis of Lactose Monolaurate as Influenced by Various Lipases and Solvents. Journal of Molecular Catalysis B: Enzymatic, 60, 171-177. https://doi.org/10.1016/j.molcatb.2009.05.003

[9] do Neta, N.A.S., Santos, J.C.S., de Sancho, S.O., Rodrigues, S., Gonçalves, L.R.B., Rodrigues, L.R. and Teixeira, J.A. (2012) Enzymatic Synthesis of Sugar Esters and Their Potential as Surface-Active Stabilizers of Coconut Milk Emulsions. Food Hydrocolloids, 27, 324-331. https://doi.org/10.1016/j.foodhyd.2011.10.009

[10] Chiralt, A. (2005) Food Emulsions. Food Engineering, Vol II. Oxford Press, Oxford. http://www.eolss.net/sample-chapters/c10/E5-10-03-05.pdf

[11] McClements, D.J. (2005) Food Emulsions: Principles, Practices, and Techniques. CRC Press, Boca Raton.

[12] Stability of Various Beverage Emulsions (2009) Food Application Paper. 1-7. http://www.titanex.com.tw/doc/tecsupport/ANF-Turbiscan-application $\% 20$ paper\% 20on\%20\%20beverage\%20emulsions.pdf

[13] Bai, L. and McClements, D.J. (2016) Extending Emulsion Functionality: Post-Homogenization Modification of Droplet Properties. Processes, 4, 2-18. https://doi.org/10.3390/pr4020017

[14] Trujillo-Cayado, L.A., Natera, A., García, M.C., Muñoz, J. and Alfaro, M.C. (2015) Rheological Properties and Physical Stability of Ecological Emulsions Stabilized by a Surfactant Derived from Cocoa Oil and High Pressure Homogenization. Grasas $y$ Aceites, 66, e087. https://doi.org/10.3989/gya.1313143

[15] Kaombe, D.D., Lenes, M., Toven, K. and Glomm, W.R. (3013) Turbiscan as a Tool for Studying the Phase Separation Tendency of Pyrolysis Oil. Energy \& Fuels, 27, 1446-1452. https://doi.org/10.1021/ef302121r

[16] Huck-Iriart, C., Candal, R.J. and Herrera, M.L. (2011) Effect of Processing Conditions and Composition on Sodium Caseinate Emulsions Stability. Procedia Food Science, 1, 116-122. https://doi.org/10.1016/j.profoo.2011.09.019 
[17] Garg, N., Martini, S., Britt, D.W. and Walsh, M.K. (2010) Emulsifying Properties of Lactose-Amines in Oil-in-Water Emulsions. Food Research International, 43, 1111-1115. https://doi.org/10.1016/j.foodres.2010.02.003

[18] Beckman Coulter (2011) Instruction for Use LS 13320 Laser Diffraction Particle Size Analyzer. Beckman Coulter Inc. https://www.beckmancoulter.com/wsrportal/techdocs?docname=B05577AB.pdf

[19] Szüts, A. and Szabó-Révész, P. (2012) Sucrose Esters as Natural Surfactants in Drug Delivery Systems-A Mini-Review. International Journal of Pharmaceutics, 433, 1-9.

[20] Patist, A., Bhagwat, S.S., Penfield, K.W., Aikens, P. and Shah, D.O. (2000) On the Measurement of Critical Micelle Concentrations of Pure and Technical-Grade Nonionic Surfactants. Journal of Surfactants and Detergents, 3, 53-58. https://doi.org/10.1007/s11743-000-0113-4

[21] Suradkar, Y.R. and Bhagwat, S.S. (2006) CMC Determination of an Odd Carbon Chain Surfactant $\left(\mathrm{C}_{13} \mathrm{E}_{20}\right)$ Mixed with Other Surfactants Using a Spectrophotometric Technique. Journal of Chemical \& Engineering Data, 51, 2026-2031. https://doi.org/10.1021/je060064a

[22] Hait, S.K. and Moulik, S.P. (2001) Determination of Critical Micelle Concentration (CMC) of Nonionic Surfactants by Donor-Acceptor Interaction with Iodine and Correlation of CMC with Hydrophile-Lipophile Balance and Other Parameters of the Surfactants. Journal of Surfactants and Detergents, 4, 303-309. https://doi.org/10.1007/s11743-001-0184-2

[23] Lee, S.-M., Sandhu, G. and Walsh, M.K. (2017) Growth Inhibitory Properties of Lactose Fatty Acid Esters. Saudi Journal of Biological Sciences, 24, 1483-1488. https://doi.org/10.1016/j.sjbs.2015.10.013

[24] Wagh, A., Shen, S., Shen, F.A., Miller, C.D. and Walsh, M.K. (2012) Investigating the Effect of Lactose Monolaurate on Pathogenic and Non-Pathogenic Bacteria. Applied Environmental Microbiology, 78, 3465-3468. https://doi.org/10.1128/AEM.07701-11

[25] Chen, Y., Nummer, B. and Walsh, M.K. (2014) Anti-Listerial Activity of Lactose Monolaurate in Milk, Drinkable Yogurt and Cottage Cheese. Letters in Applied Microbiology, 58, 156-162. https://doi.org/10.1111/lam.12169

[26] El Rassi, Z. (1995) Carbohydrate Analysis: High Performance Liquid Chromatography and Capillary Electrophoresis. Elsevier, Amsterdam, 672.

[27] Ritthitham, S. (2009) Synthesis of Sucrose Fatty Acid Esters as Catalyzed by Alkaline Protease AL 89 and Candida antarctica Lipase B in Hydrophilic Solvents. Department of Chemistry and Bioscience, Aalborg University, Aalborg.

[28] Tadros, T.F. (2013) Emulsion Formation and Stability. Wiley Online Library.

[29] Whitehurst, R.J. (2004) Emulsifiers in Food Technology. Blackwell Publishing Ltd., Hoboken.

[30] Smith, J. (1991) Food Additive User's Handbook. Springer, Berlin.

[31] Pawlik, A., Kurukji, D., Norton, I. and Spyropoulos, F. (2016) Food-Grade Pickering Emulsions Stabilised with Solid Lipid Particles. Food \& Function, 7, 2712-2721. https://doi.org/10.1039/C6FO00238B

[32] Giulietti, M. and Bernardo, A. (2012) Crystallization by Antisolvent Addition and Cooling, Crystallization-Science and Technology. InTech, London.

[33] Rao, J. and McClements, D.J. (2011) Food-Grade Microemulsions, Nanoemulsions and Emulsions: Fabrication from Sucrose Monopalmitate \& Lemon Oil. Food Hydrocolloids, 25, 1413-1423. https://doi.org/10.1016/j.foodhyd.2011.02.004 